\title{
Chapter 25 \\ Impact of Respiratory-Gated FMISO-PET/CT for the Quantitative Evaluation of Hypoxia in Non-small Cell Lung Cancer
}

\author{
Shiro Watanabe, Kenji Hirata, Shozo Okamoto, and Nagara Tamaki
}

\begin{abstract}
Hypoxia is present in various solid tumors, including non-small cell lung cancer (NSCLC) and is associated with treatment resistance and poor prognosis. ${ }^{18}$ F-Fluoromisonidazole (FMISO) is a major PET tracer for hypoxia imaging. Previous studies have evaluated the potential role of FMISO-PET as a prognostic tool and assessed tumor reoxygenation following nonsurgical treatment in NSCLC. However, for cancers located in the thorax or abdomen, the patient's breathing causes motion artifacts and misregistration between PET and CT images. PET/CT with the respiratory-gating technique improves the measurement of lesion uptake and tumor volume. We investigated the usefulness of respiratory gating for FMISOPET/CT-based quantification of hypoxia. Among the 14 patients examined, hypoxia was observed in three patients with non-gated acquisition and in five patients with respiratory gating. The SUVmax, tumor-to-muscle ratio, tumor-to-blood ratio, and hypoxic volume were statistically significantly higher in respiratory-gated (RG) images than in non-respiratory-gated (NG) images. RG FMISO-PET/CT may be useful for the accurate quantification of hypoxia.
\end{abstract}

Keywords Non-small cell lung cancer • Hypoxia • FMISO • Respiratory gating

\subsection{Background}

Lung cancer is one of the most common cancers and is the leading cause of cancer death worldwide. Although survival rates have improved in non-small cell lung cancer (NSCLC), the long-term outcome remains poor compared with other cancers. Locoregional failure is not rare, particularly after chemoradiotherapy, and may be attributed to intrinsic tumor resistance to radiotherapy and/or chemotherapy

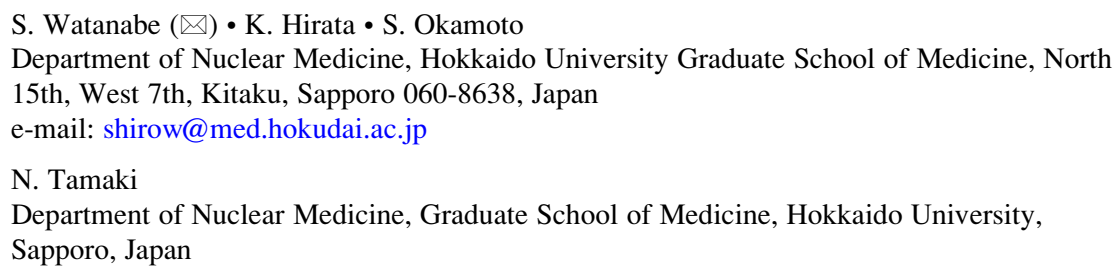




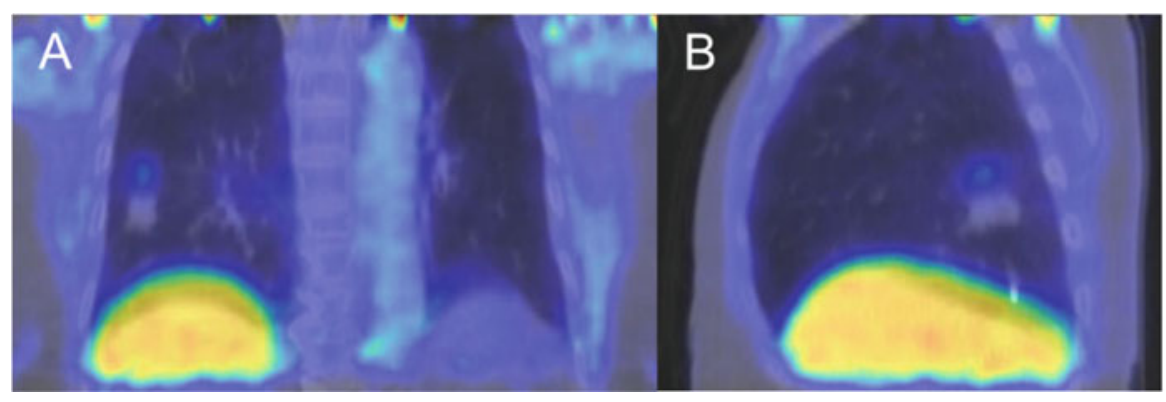

Fig. 25.1 Coronal (a) and sagittal (b) sections of thorax of a NSCLC patient in FMISO-PET/CT. The PET scan shows significant blurring and misregistration of a malignant lung lesion and the liver boundary compared with the CT scan

[1]. Intratumoral hypoxia accelerates radioresistance and chemoresistance, and thus hypoxic tumors require a 2.5-3 times radiotherapy dose to achieve the same cytotoxic effect [2]. Hypoxia may also promote metastatic spread [3].

${ }^{18} \mathrm{~F}$-Fluoromisonidazole (FMISO) is a major PET tracer for hypoxia imaging. The combination of positron emission tomography (PET) and computed tomography (CT) is valuable in cancer diagnosis, follow-up, and treatment management. Previous studies have evaluated the potential role of FMISO-PET as a prognostic tool and in the assessment of the presence of tumor reoxygenation following nonsurgical treatment of NSCLC $[1,4]$.

However, if the tumor is located in the thorax or abdomen, the patient's breathing causes motion artifacts, resulting in misregistration between PET and CT images (Fig. 25.1) [5]. Because CT is used for attenuation correction of PET images, such misregistration affects image reconstruction. The patient's breathing leads to marked displacement of most of the internal organs, from the apical region of the lungs down to the abdominal organs. Internal organ movement has a degrading effect on image quality and quantitative values in terms of spatial resolution and contrast [6]. Respiratory gating is a technique for improving the measurement of lesion uptake and tumor volume in PET/CT [7]. Motion management is becoming an important issue in both diagnostic and therapeutic applications. A series of studies in ${ }^{18} \mathrm{~F}$ fluorodeoxyglucose PET/CT have shown that respiratory-gated (RG) 4D-PET/CT and breath-holding protocols allow compensation for image degradation and artifacts induced by respiratory movements [6]. In contrast, there has been no study in which RG FMISO-PET/CT was evaluated. We investigated the usefulness of respiratory gating in FMISO-PET/CT-based quantification of hypoxia.

\subsection{Materials and Methods}

\subsubsection{Subjects}

We examined 14 patients [8 men, 6 women; median age (range) 78 (50-90) year] with pretreatment stages I-III NSCLC (Table 25.1). None of the patients had ever 
Table 25.1 Patient characteristics

\begin{tabular}{l|l}
\hline Characteristic & Number/value \\
\hline Male (Female) & $8(6)$ \\
\hline Median age (range) [year] & $78(50-90)$ \\
\hline Administered FMISO activity [MBq] & $397.6 \pm 15.7$ \\
\hline T stage & \\
\hline I & 8 \\
\hline II & 5 \\
\hline III & 1 \\
\hline Tumor length (range) $[\mathrm{mm}]$ & $29.3(12.0-53.8)$ \\
\hline
\end{tabular}

received radiotherapy. The respiratory status of the patients was not considered as an exclusion criterion. All these patients gave their written informed consent to participate in this study. This study was approved by the Institutional Review Board of Hokkaido University.

\subsubsection{FMISO-PET/CT Studies}

PET images were acquired using a whole-body time-of-flight PET/CT scanner (GEMINI-TF; Philips). We administered $400 \mathrm{MBq}$ of ${ }^{18}$ F-FMISO intravenously. Four hours after injection, static emission scans with the field of view covering the entire thorax were obtained in the 3D mode. Our protocol included a 4D CT scan and a 30-min list-mode PET acquisition in one bed position centered on the primary tumor. Respiratory signals were detected using a respiratory monitor system (Philips Bellows) with a length sensor in a belt strapped around the patient's upper abdomen.

The PET scanning protocol is shown in Fig. 25.2. To reconstruct RG images, the respiratory cycle was divided into five phases of the same duration. The third phase, which corresponds to expiration, was used for reconstruction. Non-respiratorygated (NG) images were reconstructed with $6 \mathrm{~min}$ of acquisition of PET data (i.e., sub-dataset of 12-18 $\mathrm{min}$ were extracted from the complete dataset of $30 \mathrm{~min}$ ). For all PET image reconstructions, photon attenuation was corrected using 4D CT images. Reconstructions were performed using 3D-RAMLA (ordered subset expectation maximization).

\subsubsection{Image Analysis}

FMISO uptake $4 \mathrm{~h}$ after injection was quantified using (1) standardized uptake values ( $\mathrm{SUV}=1 \mathrm{~g} / \mathrm{mL} \mathrm{X}$ measured radioactivity $\mathrm{X}$ body weight/injected radioactivity), (2) tumor-to-muscle ratio (TMR), and (3) tumor-to-blood ratio (TBR). Paraspinal muscles were used as the reference muscle. Venous blood was sampled immediately before the PET/CT scanning and counted for radioactivity 


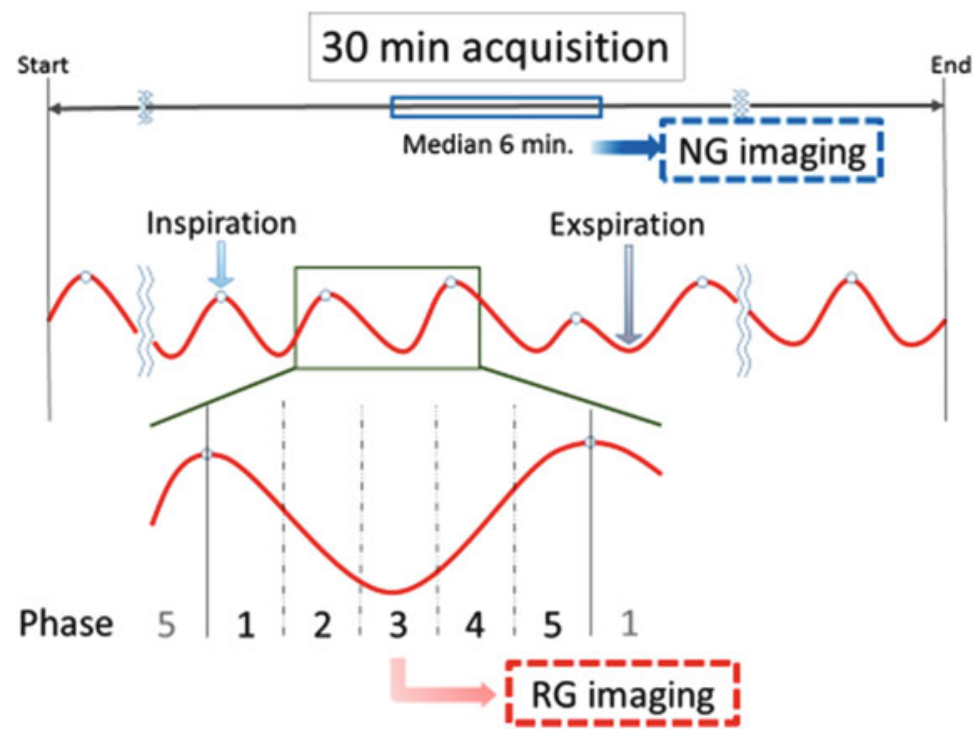

Fig. 25.2 Summary of the PET scan protocol. All the acquisitions lasted $30 \mathrm{~min}$. For respiratorygated imaging, the third phase, which corresponds to expiration, was used for reconstruction. Nonrespiratory-gated images were reconstructed with 6 min of acquired PET data (i.e., 12-18 $\mathrm{min}$ )

using a cross-calibrated well counter. We also calculated hypoxic volume (HV) as an area TBR higher than 1.5 [8]. Patients having nonzero HV were considered as having hypoxic tumor. Differences in SUVmax, TMR, TBR, and HV between RG and NG images were statistically analyzed for significance.

\subsubsection{Statistical Analysis}

All results are expressed here as mean \pm standard deviation (SD). A statistical paired $t$-test was employed to evaluate the statistical significance of the differences in SUVmax, TMR, and TBR between RG and NG. HV was compared between RG and NG images using the Wilcoxon signed-rank test because of the non-normal distribution of $\mathrm{HV}$. P values smaller than 0.05 were considered statistically significant.

\subsection{Results and Discussion}

In all the 14 patients, the tumor was visually identifiable from its higher signal intensities than the surrounding lung tissues (Figs. 25.3 and 25.4). Quantitatively, SUVmax, TMR, and TBR were all significantly higher on RG images $(1.93 \pm 1.11$, 

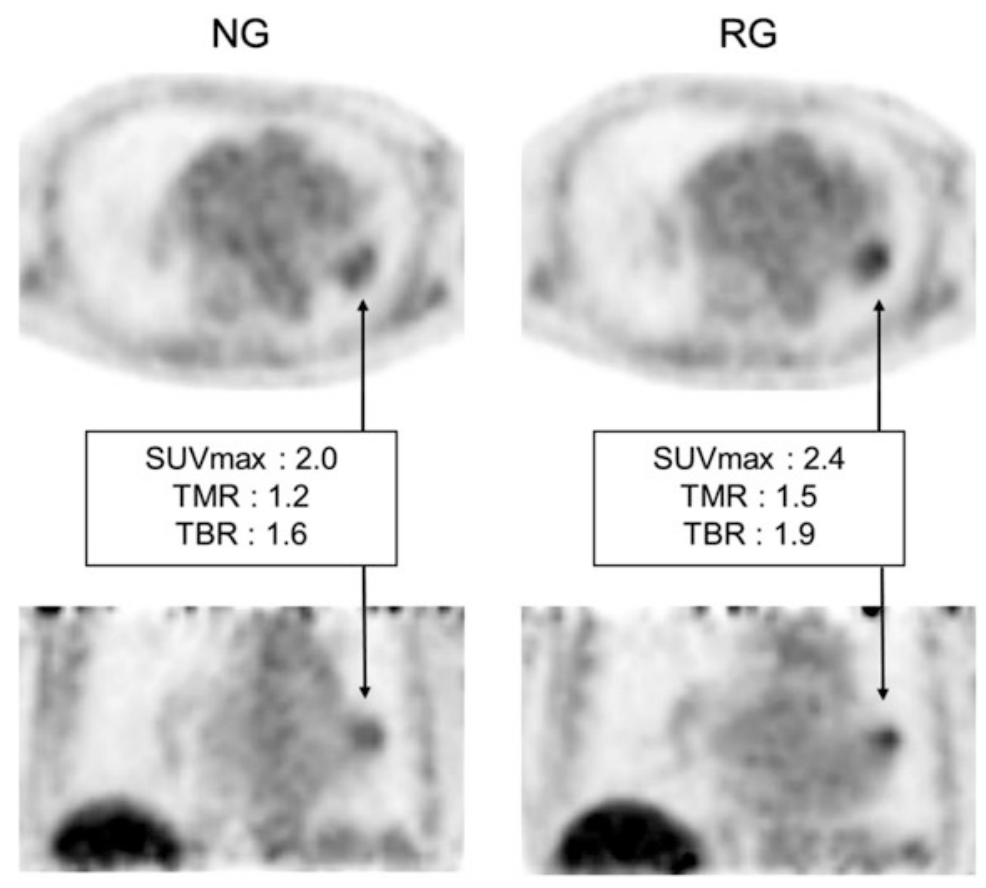

Fig. 25.3 Axial and coronal sections of NG and RG PET images of a patient with a NSCLC lesion in the left lower lobe. In addition to the difference in morphological appearances of the lesion between the NG and RG images, there are considerable increases in SUVmax, TMR, and TBR

$1.46 \pm 0.78$, and $1.42 \pm 0.87$, respectively) than on NG images $(2.09 \pm 1.11$, $1.61 \pm 0.78$, and $1.53 \pm 0.87$, respectively) (Table 25.2, Fig. 25.5).

Whereas the NG images showed tumor hypoxia in three patients, the RG images identified tumor hypoxia in two more patients (i.e., a total of 5 patients). In patients with hypoxia, HV on NG images was $12.8 \pm 22.6$, whereas that on RG images was $13.2 \pm 22.7$, which was significantly higher (Table 25.3).

The results of this study showed significant differences in various quantitative values between RG and NG. Theoretically, RG is less affected by motion artifacts, and thus the images acquired with RG are considered to be more accurate than those with NG. Our data suggest the risk of using non-respiratory gating for FMISO PET in NSCLC, because non-respiratory gating could significantly underestimate tumor hypoxia. Instead, the use of respiratory gating is recommended as a standard technique for treatments targeting a hypoxic region.

The ability to determine the degree and extent of hypoxia in NSCLC is not only important prognostically but also in the selection of candidate patients for hypoxiamodifying treatments. [9] Among different treatments, radiotherapy would most benefit from hypoxia imaging techniques. Radiobiological modeling suggests that hypoxia would have a greater impact on the efficacy of a single-large-fraction treatment than on that of fractionated treatment because of the lack of 


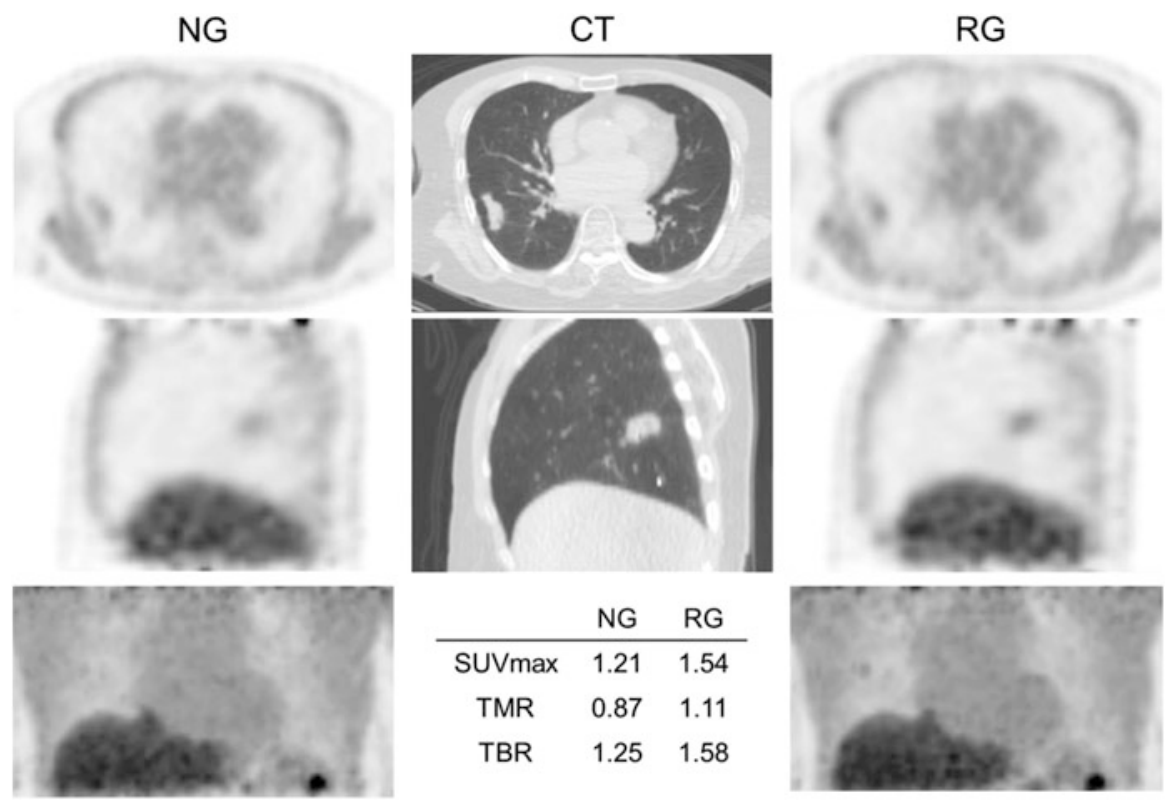

Fig. 25.4 Images of an 87-year-old female with stage II NSCLC in the right lower lobe. In nonrespiratory-gated maximum intensity projection (MIP) imaging, no primary lesion was detected. However, in respiratory-gated MIP imaging, the lesion was visually detected

Table 25.2 SUVmax, TMR, and TBR of lesions on RG and NG images

\begin{tabular}{l|l|l|l|l|l|l}
\hline & \multicolumn{3}{|l|}{ SUVmax } & \multicolumn{2}{l|}{ TMR } & \multicolumn{2}{l}{ TBR } \\
\hline & NG & RG & NG & RG & NG & RG \\
\hline Mean & 1.93 & 2.09 & 1.46 & 1.61 & 1.42 & 1.53 \\
\hline SD & 1.11 & 1.11 & 0.78 & 0.78 & 0.87 & 0.86 \\
\hline
\end{tabular}

Abbreviations: $N G$ non-gating, $R G$ respiratory gating, $S D$ standard deviation, SUVmax maximum standardized uptake value, $T B R$ tumor-to-blood ratio, TMR tumor-to-muscle ratio

reoxygenation in the former [9]. Information on tumor hypoxia may be used to modify the radiation planning, especially the treatment fraction, to maximize cytotoxic effects.

However, as mentioned above, respiratory motion during PET image quantification can introduce image misregistration errors, and if uncorrected images are acquired, such errors may eventually hinder adequate patient management [10]. As a combined treatment strategy with functional information provided by PET imaging, correction of PET images for respiratory motion artifacts may increase the efficacy of individually tailored therapy. If FMISO-PET imaging predicts local failure, then it can be used for guiding the selection of patients who would benefit from dose escalation, modification of fractionation, or additional treatment with a hypoxic cell radiosensitizer. 


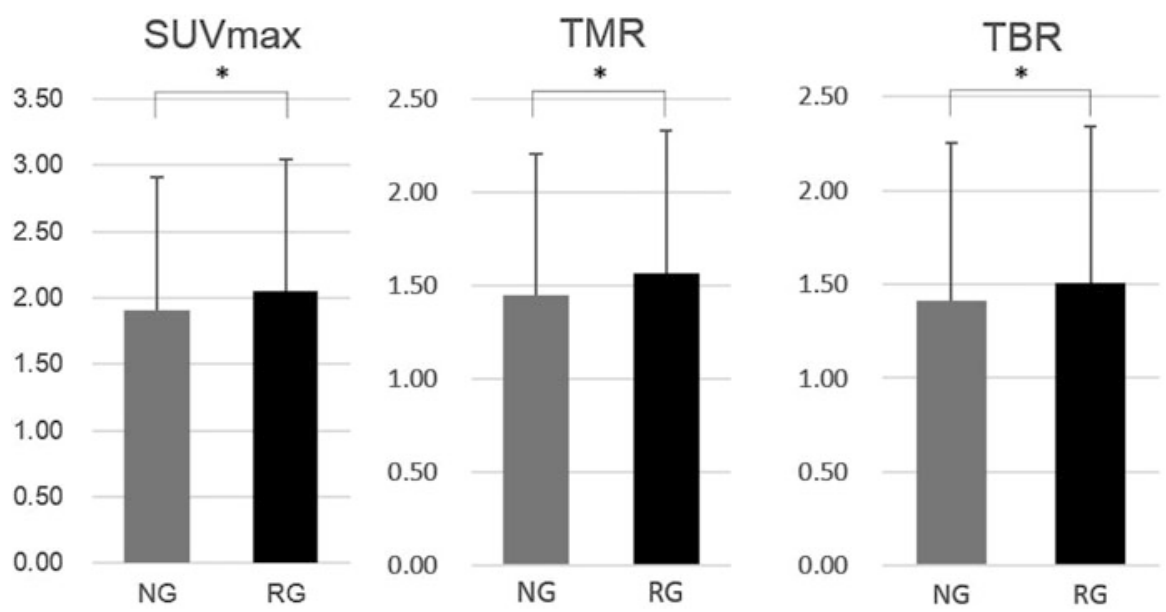

Fig. 25.5 SUVmax, TMR, and TBR were significantly higher on RG images than on NG images $(* p<0.05$, paired $t$-test $)$

Table 25.3 Increase in HV with RG

\begin{tabular}{l|l|l|c}
\hline Age & Sex & HV in NG & HV in RG \\
\hline 72 & Male & 0 & 0.19 \\
\hline 50 & Male & 9.15 & 9.92 \\
\hline 62 & Female & 52.54 & 53.12 \\
\hline 85 & Male & 2.05 & 2.69 \\
\hline 81 & Male & 0.00 & 0.13 \\
\hline Mean & & 12.8 & 13.2 \\
\hline SD & & 22.6 & 22.7 \\
\hline
\end{tabular}

Abbreviations: $H V$ hypoxic volume, $N G$ non-gating, $R G$ respiratory gating, $S D$ standard deviation

One of the limitations of our study was the relatively small number of patients examined. Further clinical study will be required to clarify the diagnostic value of the quantitative evaluation of hypoxia with RG in association with local recurrence and prognosis.

\subsection{Conclusion}

Respiratory gating in FMISO-PET/CT could provide higher sensitivity of hypoxic evaluation and accurate quantification of hypoxia. 
Open Access This chapter is distributed under the terms of the Creative Commons AttributionNoncommercial 2.5 License (http://creativecommons.org/licenses/by-nc/2.5/) which permits any noncommercial use, distribution, and reproduction in any medium, provided the original author(s) and source are credited.

The images or other third party material in this chapter are included in the work's Creative Commons license, unless indicated otherwise in the credit line; if such material is not included in the work's Creative Commons license and the respective action is not permitted by statutory regulation, users will need to obtain permission from the license holder to duplicate, adapt or reproduce the material.

\section{References}

1. Yip C, Blower PJ, Goh V, Landau DB, Cook GJ. Molecular imaging of hypoxia in non-smallcell lung cancer. Eur J Nucl Med Mol Imaging. 2015;42(6):956-76. doi:10.1007/s00259-0153009-6.

2. Gray LH, Conger AD, Ebert M, Hornsey S, Scott OC. The concentration of oxygen dissolved in tissues at the time of irradiation as a factor in radiotherapy. Br J Radiol. 1953;26 (312):638-48. doi:10.1259/0007-1285-26-312-638.

3. Gilkes DM, Semenza GL, Wirtz D. Hypoxia and the extracellular matrix: drivers of tumour metastasis. Nat Rev Cancer. 2014;14(6):430-9. doi:10.1038/nrc3726.

4. Cherk MH, Foo SS, Poon AM, Knight SR, Murone C, Papenfuss AT, et al. Lack of correlation of hypoxic cell fraction and angiogenesis with glucose metabolic rate in non-small cell lung cancer assessed by 18F-Fluoromisonidazole and 18F-FDG PET. J Nucl Med Off Publ Soc Nucl Med. 2006;47(12):1921-6.

5. Callahan J, Kron T, Schneider-Kolsky M, Hicks RJ. The clinical significance and management of lesion motion due to respiration during PET/CT scanning. Cancer Imaging Off Publ Int Cancer Imaging Soc. 2011;11:224-36. doi:10.1102/1470-7330.2011.0031.

6. Bettinardi V, Picchio M, Di Muzio N, Gilardi MC. Motion management in positron emission tomography/computed tomography for radiation treatment planning. Semin Nucl Med. 2012;42(5):289-307. doi:10.1053/j.semnuclmed.2012.04.001.

7. Jani SS, Robinson CG, Dahlbom M, White BM, Thomas DH, Gaudio S, et al. A comparison of amplitude-based and phase-based positron emission tomography gating algorithms for segmentation of internal target volumes of tumors subject to respiratory motion. Int J Radiat Oncol Biol Phys. 2013;87(3):562-9. doi:10.1016/j.ijrobp.2013.06.2042.

8. Okamoto S, Shiga T, Yasuda K, Ito YM, Magota K, Kasai K, et al. High reproducibility of tumor hypoxia evaluated by $18 \mathrm{~F}$-fluoromisonidazole PET for head and neck cancer. J Nucl Med Off Publ Soc Nucl Med. 2013;54(2):201-7. doi:10.2967/jnumed.112.109330.

9. Meng X, Kong FM, Yu J. Implementation of hypoxia measurement into lung cancer therapy. Lung Cancer (Amsterdam, Netherlands). 2012;75(2):146-50. doi:10.1016/j.lungcan.2011.09. 009.

10. Grootjans W, de Geus-Oei LF, Meeuwis AP, van der Vos CS, Gotthardt M, Oyen WJ, et al. Amplitude-based optimal respiratory gating in positron emission tomography in patients with primary lung cancer. Eur Radiol. 2014;24(12):3242-50. doi:10.1007/s00330-014-3362-z. 